九州大学学術情報リポジトリ

Kyushu University Institutional Repository

\title{
Philosophical Properites of Euler's Formula in its Application to Symbolic Representation of Some Aspects of Ruminant Agriculture
}

Shimojo, Masataka

Laboratory of Animal Feed Science, Division of Animal Science, Department of Animal and Marine Bioresoruce Sciences, Faculty of Agriculture, Kyushu University

Ikeda, Kentaro

Research Fellow, Faculty of Agriculture, Kyushu University

Asano, Yoki

Research Fellow, Faculty of Agriculture, Kyushu University

Ishiwaka, Reiko

他

https://doi.org/10.5109/4529

出版情報：九州大学大学院農学研究院紀要. 48 (1/2)，pp.77-83，2003-10-01. Faculty of Agriculture, Kyushu University

バージョン :

権利関係 : 


\title{
Philosophical Properties of Euler's Formula in its Application to Symbolic Representation of Some Aspects of Ruminant Agriculture
}

\author{
Masataka SHIMOJO ${ }^{\dagger}$, Kentaro IKEDA ${ }^{1)}$, Yoki ASANO ${ }^{1)}$, Reiko ISHIWAKA ${ }^{1)}$, \\ Tao SHAO $^{2)}$, Hiroyuki SATO ${ }^{3)}$, Manabu TOBISA, Yutaka NAKANO ${ }^{4}$, \\ Noriko OHBA $^{5}$, Yasukatsu YANO $^{6)}$ and Yasuhisa MASUDA \\ Laboratory of Animal Feed Science, Division of Animal Science, Department of \\ Animal and Marine Bioresource Sciences, Faculty of Agriculture, \\ Kyushu University, Fukuoka 812-8581, Japan \\ (Received June 30, 2003 and accepted July 15, 2003)
}

This study suggests that philosophical properties of Euler's formula make some contribution to symbolic descriptions of some micro- and macro-structures in ruminant agriculture.

\section{INTRODUCTION}

Using Euler's formula some aspects of ruminant agriculture were described symbolically; field-forage-ruminant-relationships suggesting the cycling of matter in a narrow sense (Shimojo et al., 2003a, b), exponential functions with base $e$ used for the growth analysis of ruminants and forages (Shimojo et al., 2003c), and spiral structures showing topological similarities to micro-structures in ruminants and forages (Shimojo et al., 2003d). Euler's formula is used for descriptions of various natural phenomena (Yoshida, 2000). What property of Euler's formula is involved in the symbolic description of these three different things?

The present study was designed to investigate philosophical properties of Euler's formula in the application to symbolic descriptions of some aspects of ruminant agriculture.

\section{PHILOSOPHICAL PROPERTIES OF EULER'S FORMULA}

\section{A complex representation of ' 1 ' and its properties}

A complex representation of ' 1 ' is given by

1) Research Fellow, Faculty of Agriculture, Kyushu University

2) Visiting Research Scientist from Lanzhou Institute of Animal Science and Veterinary Medicine of CAAS, Lanzhou Gansu Province of the People's Republic of China

3) Laboratory of Animal Feed Science, Division of Animal Science, Department of Animal and Marine Bioresource Sciences, Graduate School of Bioresource and Bioenvironmental Sciences, Kyushu University

4) Kyushu University Farm, Fukuoka 811-2307

5) Research Student, School of Agriculture, Kyushu University

6) Former Technical Specialist

+ Corresponding author (E-mail: mshimojo@agr.kyushu-u.ac.jp) 


$$
\begin{aligned}
1 & =\boldsymbol{i} \cdot(-\boldsymbol{i}) \\
& =(\cos \gamma+i \sin \gamma) \cdot(\sin \gamma+i \cos \gamma) \cdot(\cos \gamma-i \sin \gamma) \cdot(\sin \gamma-i \cos \gamma),
\end{aligned}
$$

where $i=$ imaginary unit, $0<\gamma<\pi / 2,(\cos \gamma+i \sin \gamma) \cdot(\sin \gamma+i \cos \gamma)=i,(\cos \gamma-i \sin \gamma) \cdot(\sin \gamma$ $-\boldsymbol{i} \cos \gamma)=-\boldsymbol{i}$.

The equality (1) shows a kind of order of transformation of ' 1 ': real number $\rightarrow$ imaginary number $\rightarrow$ complex number. This suggests that the complex number exists at deeper level than the real number. It is also shown in (1) that $\cos \gamma+\boldsymbol{i} \sin \gamma=\exp (\boldsymbol{i} \gamma)$ is Euler's formula and $\cos \gamma-\boldsymbol{i} \sin \gamma=\exp (-\boldsymbol{i} \gamma)$ is its conjugate complex, suggesting an importance of complex representation of ' 1 '.

There is another way of complex representation of ' 1 '. Thus,

$$
\begin{aligned}
1 & =\{-(\cos \gamma+i \sin \gamma)\} \cdot\{-(\sin \gamma+i \cos \gamma)\} \cdot\{-(\cos \gamma-i \sin \gamma)\} \cdot\{-(\sin \gamma-i \cos \gamma)\} \\
& =(-\cos \gamma+i \sin \gamma) \cdot(-\sin \gamma+i \cos \gamma) \cdot(-\cos \gamma-i \sin \gamma) \cdot(-\sin \gamma-i \cos \gamma),
\end{aligned}
$$

where $(-\cos \gamma+i \sin \gamma) \cdot(-\sin \gamma+i \cos \gamma)=-\boldsymbol{i},(-\cos \gamma-i \sin \gamma) \cdot(-\sin \gamma-i \cos \gamma)=\boldsymbol{i}$.

Replacing plus sign of the real part in (1) with minus sign gives (2). Thus, the equality (2) is a reflection of (1) in the imaginary axis. Replacing plus (minus) sign of the imaginary part in (1) with minus (plus) sign gives (1) itself, a symmetric property.

Four components in (1) cannot be separated because they are united by multiplication, and the same rule applies to four components in (2). This was not mentioned in our previous reports (Shimojo et al., 2003a, d), which gave a misunderstanding to the construction of the following two sequences.

$$
\begin{aligned}
& (\cos \gamma+i \sin \gamma) \stackrel{\times i}{\longrightarrow}(-\sin \gamma+i \cos \gamma) \stackrel{\times i}{\longrightarrow}(-\cos \gamma-i \sin \gamma) \stackrel{\times i}{\longrightarrow}(\sin \gamma-i \cos \gamma), \\
& (\sin \gamma+i \cos \gamma) \stackrel{\times(-i)}{\longrightarrow}(\cos \gamma-i \sin \gamma) \stackrel{\times(-i)}{\longrightarrow}(-\sin \gamma-i \cos \gamma) \stackrel{\times(-i)}{\longrightarrow}(-\cos \gamma+i \sin \gamma) .
\end{aligned}
$$

In (3) and (4) eight components are separated in order to construct new sequences. Broadly speaking, taking up two ways of complex representation of ' 1 ' [(1) and (2)] is considered just a means to get the two sequences (3) and (4). In other words, (3) and (4) can be obtained even if there is not the description of (1) and (2). It seems, however, that (3) and (4) have relationships to (1) and (2), which will be discussed in the section after next.

The sequence (3) shows a series of $\pi / 2$ rotations [ $\times \boldsymbol{i}$ ] of Euler's formula: anti-clockwise rotations in the planar representation, and right-handed spirals with phase shifts in the stereographic representation. The sequence (4) shows a series of $-\pi / 2$ rotations [ $X$ $(-\boldsymbol{i})$ ] of the conjugate complex to Euler's formula: clockwise rotations in the planar representation, and left-handed spirals with phase shifts in the stereographic representation. Therefore, these two show inverse properties each other.

\section{Combining (1) and (2), combining (3) and (4), and distribution of the eight components on the complex plane}

The combination of (1) and (2) is given by the product of them. Thus,

$$
\begin{aligned}
1= & (\cos \gamma+i \sin \gamma) \cdot(-\sin \gamma+i \cos \gamma) \cdot(-\cos \gamma-i \sin \gamma) \cdot(\sin \gamma-i \cos \gamma) \\
& \cdot(\sin \gamma+i \cos \gamma) \cdot(\cos \gamma-i \sin \gamma) \cdot(-\sin \gamma-i \cos \gamma) \cdot(-\cos \gamma+i \sin \gamma)
\end{aligned}
$$


The combination of (3) and (4) is given by the addition of them. Thus,

$$
\begin{aligned}
& (\cos \gamma+i \sin \gamma)+(-\sin \gamma+i \cos \gamma)+(-\cos \gamma-i \sin \gamma)+(\sin \gamma-i \cos \gamma) \\
& +(\sin \gamma+i \cos \gamma)+(\cos \gamma-i \sin \gamma)+(-\sin \gamma-i \cos \gamma)+(-\cos \gamma+i \sin \gamma)=0 .
\end{aligned}
$$

The eight components are designated as follows:

$$
\begin{aligned}
& \cos \gamma+i \sin \gamma=\mathrm{A}, \quad \sin \gamma+\boldsymbol{i} \cos \gamma=\mathrm{B}, \quad-\sin \gamma+\boldsymbol{i} \cos \gamma=\mathrm{C}, \quad-\cos \gamma+i \sin \gamma=\mathrm{D}, \\
& -\cos \gamma-i \sin \gamma=\mathrm{E}, \quad-\sin \gamma-i \cos \gamma=\mathrm{F}, \quad \sin \gamma-i \cos \gamma=\mathrm{G}, \quad \cos \gamma-i \sin \gamma=\mathrm{H} .
\end{aligned}
$$

The plotting of them on the complex plane shows the following distribution (Fig. 1): A and $\mathrm{B}$ are located in the 1st quadrant, $\mathrm{C}$ and $\mathrm{D}$ in the 2nd quadrant, $\mathrm{E}$ and $\mathrm{F}$ in the 3rd quadrant, and $\mathrm{G}$ and $\mathrm{H}$ in the 4 th quadrant. In addition, important relationships between the eight components are as follows: $A+E=0, \quad B+F=0, \quad C+G=0, \quad D+H=0$.

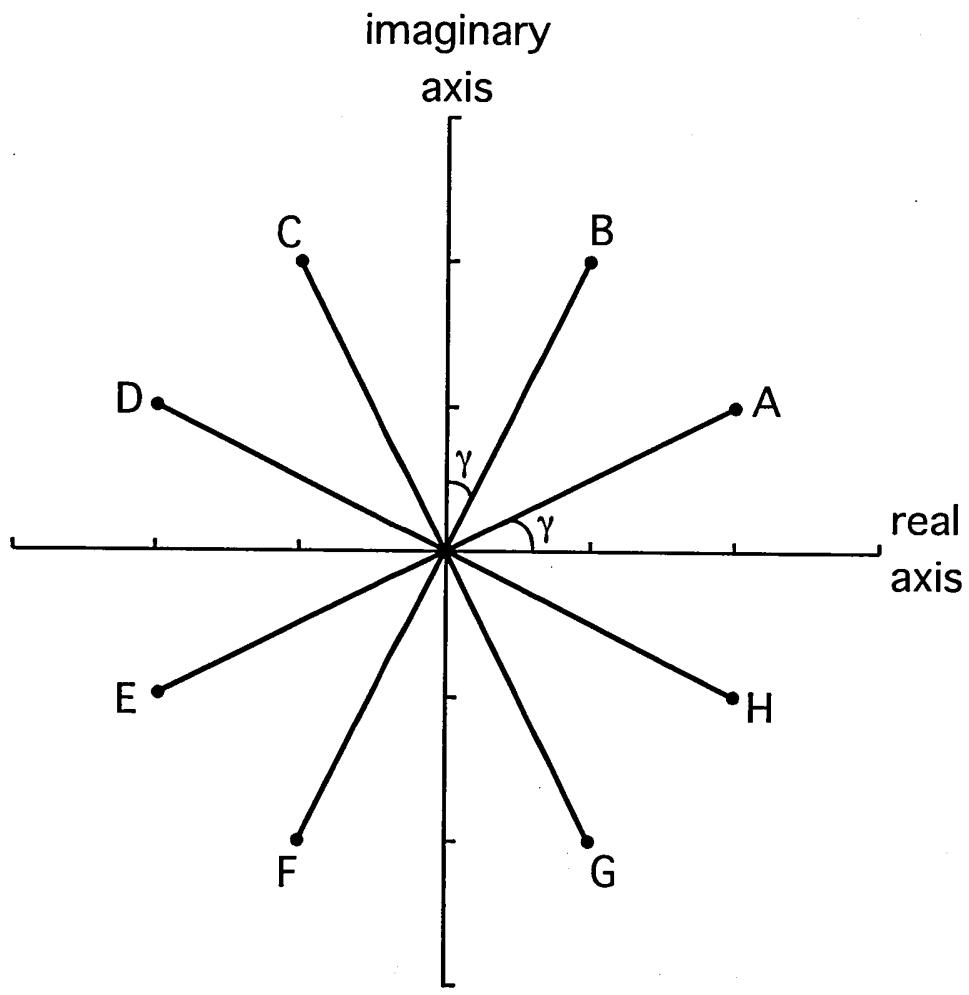

Fig. 1. An illustration of eight components on the complex plane $(A=\cos \gamma+$ $i \sin \gamma, \mathrm{B}=\sin \gamma+i \cos \gamma, \mathrm{C}=-\sin \gamma+i \cos \gamma, \mathrm{D}=-\cos \gamma+i \sin \gamma, \mathrm{E}=-\cos \gamma$ $-i \sin \gamma, \mathrm{F}=-\sin \gamma-\boldsymbol{i} \cos \gamma, \mathrm{G}=\sin \gamma-\boldsymbol{i} \cos \gamma, \mathrm{H}=\cos \gamma-\boldsymbol{i} \sin \gamma)$. The product of them gives ' 1 ' and the addition of them gives ' 0 '. 


\section{A hypothetic relationship between (5) and (6)}

The equality (5) shows that the product of eight components gives ' 1 '. The equality (6) shows that the addition of them gives ' 0 '. How will these two be related?

A hypothetic relationship between (5) and (6) might be as follows. A real number ' 1 ' has been borrowed from the seeming nothing. Therefore, this should be followed by a return of ' 1 ' to the creditor. The return of borrowed ' 1 ' might be carried out by a breakdown of the form of multiplication that unites the eight components to form ' 1 ' in (5), which results in, at that moment, a separation of them in (6). This might occur as if to cause four sets of pair appearance first, and then those of pair disappearance, where each pair is composed of a complex number and its opposite (A and E, B and F, C and G, D and $\mathrm{H})$. In addition, the stereographic representation of the eight components might also give a kind of spatial image of pair appearances and pair disappearances, if $\gamma$ takes values in order to show right-handed spirals $[A, C]$ and their opposites $[E, G]$, and left-handed spirals $[B, D]$ and their opposites $[F, H]$. Is there anything that keeps the eight components of ' 1 ' away from pair disappearances in order to be used for descriptions of some aspects of ruminant agriculture (Shimojo et al., 2003a, b, c, d)?

Since $\gamma$ takes values between 0 and $\pi / 2$ as shown in the first section, (5) and (6) will be expanded. Thus,

$$
\begin{gathered}
1_{k=1}^{n}=\prod_{k=1}^{n}\left\{\left(\cos \gamma_{k}+i \sin \gamma_{k}\right) \cdot\left(-\sin \gamma_{k}+i \cos \gamma_{k}\right) \cdot\left(-\cos \gamma_{k}-i \sin \gamma_{k}\right) \cdot\left(\sin \gamma_{k}-i \cos \gamma_{k}\right)\right. \\
\left.\cdot\left(\sin \gamma_{k}+i \cos \gamma_{k}\right) \cdot\left(\cos \gamma_{k}-i \sin \gamma_{k}\right) \cdot\left(-\sin \gamma_{k}-i \cos \gamma_{k}\right) \cdot\left(-\cos \gamma_{k}+i \sin \gamma_{k}\right)\right\} \\
\sum_{k=1}^{n}\left\{\left(\cos \gamma_{k}+i \sin \gamma_{k}\right)+\left(-\sin \gamma_{k}+i \cos \gamma_{k}\right)+\left(-\cos \gamma_{k}-i \sin \gamma_{k}\right)+\left(\sin \gamma_{k}-i \cos \gamma_{k}\right)\right. \\
\left.+\left(\sin \gamma_{k}+i \cos \gamma_{k}\right)+\left(\cos \gamma_{k}-i \sin \gamma_{k}\right)+\left(-\sin \gamma_{k}-i \cos \gamma_{k}\right)+\left(-\cos \gamma_{k}+i \sin \gamma_{k}\right)\right\}=0
\end{gathered}
$$

where $0<\gamma_{1}<\cdots<\gamma_{k}<\cdots<\gamma_{n}<\pi / 2$.

Many components ( $=8 n$ ) come from ' $1^{n}$ ' according to (7), namely $n$ sets of the eight components. However, since $1^{n}=1$, the number of components appearing from ' 1 ' might be $8 n$, and in addition, infinite if $n \rightarrow \infty$. Momentarily after a breakdown of the form of multiplication in (7), many pairs of complex number and its opposite, appear and disappear, resulting in the return of ' $1^{n}(=1)$ ' to the creditor, the seeming nothing, as shown in (8). However, these remain to be examined.

In the next chapter symbolic descriptions of some aspects of ruminant agriculture (Shimojo et al., 2003a, b, c, d) will be summarized, which is followed by suggesting what philosophical property of Euler's formula is involved in them.

\section{APPLICATION OF EULER'S FORMULA TO SYMBOLIC DESCRIPTIONS OF SOME ASPECTS OF RUMINANT AGRICULTURE}

\section{Topological similarities to micro-structures in ruminants and forages}

The stereographic representation of Euler's formula $[\exp (\boldsymbol{i} \beta)=\cos \beta+\boldsymbol{i} \sin \beta]$ using coordinates $(\beta, \cos \beta, \boldsymbol{i} \sin \beta)$ in $0<\beta$ gives spirals (Yoshida, 2000). It seems that these spirals show topological similarities to helical structures in ruminants and forages (Shimojo et al., 2003d): a helix of right-handed or left-handed property, double helix of right-handed or left-handed property, triple helix of right-handed property. The right-handed and left-handed properties are related to Euler's formula and its conjugate 
complex, respectively. The double and triple helices are related to combining Euler's formula or its conjugate complex with their phase shifts. The complementary property in the form of double helix is related to keeping the form of Euler's formula or its conjugate complex invariant with respect to their phase shifts.

\section{Obtaining exponential functions with base $e$ used for the growth analysis of ruminants and forages}

The rotation of axes of time, imaginary time $(i t)$ and real time $(t)$, in Euler's formula $[\exp (\boldsymbol{i} t)=\cos (t)+\boldsymbol{i} \sin (t)]$ gives $\exp (-t)$ when there is anti-clockwise $\pi / 2$ rotation $[\boldsymbol{i} \cdot(\boldsymbol{i} t)$ and $\boldsymbol{i} \cdot t]$, and gives $\exp (t)$ when there is clockwise $\pi / 2$ rotation $[(-\boldsymbol{i}) \cdot(\boldsymbol{i} t)$ and $(-\boldsymbol{i}) \cdot t]$. The existence of Euler's formula between $\exp (t)$ and $\exp (-t)$ suggests that two axes of time (it and $t$ intersecting orthogonally each other) separate the moving forward in time $[\exp (1 \cdot t)]$ from the moving backward in time $[\exp \{1 \cdot(-t)\}]$. However, in the actual growth analysis of ruminants and forages $\exp (-t)$ is regarded as $\exp (-1 \cdot t)$, a decay phenomenon.

Shimojo et al. (2002) showed, in a report of basic growth analysis of ruminants and forages, another type of borrowing ' 1 ' from the seeming nothing. In the definite integral of Maclaurin's series of the exponential function with base $e$, there is an occurrence of ' 0 $\rightarrow 1+(-1)$ '. This is, however, followed by the remaining of ' 1 ' and the disappearance of ' -1 ', as shown in the following calculation using $\exp (t)$ :

$$
\begin{aligned}
P & =\int_{t_{1}}^{t_{2}} \exp (t) d t \\
& =\int_{t_{1}}^{t_{2}}\left(1+\frac{t}{1 !}+\frac{t^{2}}{2 !}+\cdots+\frac{t^{n}}{n !}+\cdots \cdot\right) d t \\
& =\left[\frac{t}{1 !}+\frac{t^{2}}{2 !}+\cdots \cdots+\frac{t^{n}}{n !}+\cdots \cdots\right]_{t_{1}}^{t_{2}} \\
& =\left[\left(1+\frac{t}{1 !}+\frac{t^{2}}{2 !}+\cdots \cdots+\frac{t^{n}}{n !}+\cdots \cdot\right)-1\right]_{t_{1}}^{t_{2}} \\
& =\left[\left\{\exp \left(t_{2}\right)-1\right\}-\left\{\exp \left(t_{1}\right)-1\right\}\right] \\
& =\exp \left(t_{2}\right)-\exp \left(t_{1}\right) .
\end{aligned}
$$

The occurrence of this phenomenon is required by keeping the form of $\exp (t)$ invariant with respect to its definite integral. Another interpretation is, though this was not mentioned in our previous report (Shimojo et al., 2002), that there is only a recovering of ' 1 ' after its disappearance, therefore, '-1' has nothing to do with it. Broadly speaking at the risk of making mistakes, if the former interpretation is adopted, then the following difference will occur. There might be, in a macro-phenomenon [(9)], a kind of asymmetry in the fate between ' 1 ' and ' -1 ' after a pair appearance from the seeming nothing. This is different from micro-phenomena [(7) and (8)], where the borrowed ' 1 ' from the seeming nothing should be returned, a kind of symmetry.

\section{Application to symbolic description of field-forage-ruminant relationships}

This application is based on the following use of Euler's formula (Shimojo et al., 2003a, b). Thus, 


$$
Z_{1}=D+i I=r(\cos \theta+i \sin \theta),
$$

where $0<\theta<\pi / 2, r \cos \theta=D$ (digestible dry matter weight of the forage), $r \sin \theta=I$ (indigestible dry matter weight of the forage), $D+I=W$ (forage dry matter weight).

$Z_{1}$ is regarded as the field with standing forage composed of $D$ and $I$, because both $D$ and $I$ are considered visible due to the plus sign they have.

$$
\text { Then, } Z_{\mathbf{1}} \cdot \boldsymbol{i}=r(\cos \theta+\boldsymbol{i} \sin \theta) \cdot \boldsymbol{i}=r(-\sin \theta+\boldsymbol{i} \cos \theta),
$$

where $-r \sin \theta=-I, r \cos \theta=D . \quad Z_{1} \cdot \boldsymbol{i}$ is associated with the ruminant production from digestible nutrients of the forage, because $D$ is visible but $-I$ is invisible due to the minus sign.

$$
Z_{1} \cdot i^{2}=r(\cos \theta+i \sin \theta) \cdot i^{2}=r(-\cos \theta-i \sin \theta),
$$

where $-r \cos \theta=-D,-r \sin \theta=-I . Z_{1} \cdot i^{2}$ is regarded as the field without standing forage after harvesting for ruminant consumption, because both $-D$ and $-I$ are invisible.

$$
Z_{\mathbf{1}} \cdot \boldsymbol{i}^{3}=r(\cos \theta+\boldsymbol{i} \sin \theta) \cdot \boldsymbol{i}^{3}=r(\sin \theta-\boldsymbol{i} \cos \theta),
$$

where $r \sin \theta=I,-r \cos \theta=-D . \quad Z_{\mathbf{1}} \cdot \boldsymbol{i}^{3}$ is associated with feces excreted from the ruminant, because $I$ is visible but $-D$ is invisible.

A feature of this application is given by the following three phenomena:

$$
\begin{aligned}
& 0=r(\cos \theta+i \sin \theta)+r(-\cos \theta-i \sin \theta) \text { or } 0=(D+i I)+(-D-i I), \\
& 0=r(-\sin \theta+i \cos \theta)+r(\sin \theta-i \cos \theta) \text { or } 0=(-I+i D)+(I-\boldsymbol{i} D), \\
& 0=r(\cos \theta+i \sin \theta)+r(-\cos \theta-i \sin \theta)+r(-\sin \theta+\boldsymbol{i} \cos \theta)+r(\sin \theta-i \cos \theta) \\
& \text { or } 0=(D+i I)+(-D-\boldsymbol{i} I)+(-I+\boldsymbol{i} D)+(I-\boldsymbol{i} D) .
\end{aligned}
$$

These suggest that producing the forage and harvesting the forage form a pair through the field [(14)], animal production and feces excretion form a pair through the ruminant [(15)]. The four things form a cycle [(16)], and in addition, are of equal significance to ruminant agriculture based on the cycling of matter in a narrow sense.

\section{Suggested philosophical properties of Euler's formula in the symbolic descrip- tion of some aspects of ruminant agriculture}

Philosophical properties of Euler's formula suggested in the description of some aspects of ruminant agriculture might be the following two: (i) its symmetric properties with respect to phase shifts in the deriving not only of spirals that show topological similarities to micro-structures in ruminants and forages (Shimojo et al., 2003d) but also of the cycling of matter in a narrow sense that shows field-forage-ruminant relationships (Shimojo et al., 2003a, b), and (ii) a kind of symmetry breakdown with respect to rotations of exponent in the deriving of exponential functions with base $e$ used for the growth analysis of ruminants and forages (Shimojo et al., 2003c). These might give, therefore, rough images of some micro- and macro-structures in ruminant agriculture.

\section{REFERENCES}

Shimojo, M., Y. Asano, K. Ikeda, R. Ishiwaka, T. Shao, N. Ohba, H. Sato, Y. Matsufuji, M. Tobisa, Y. Yano and Y. Masuda 2002 Basic growth analysis and symmetric properties of exponential function with base 
e. J. Fac. Agr., Kyushu Univ., 47: 55-60

Shimojo, M., K. Ikeda, Y. Asano, R .Ishiwaka, T. Shao, H. Sato, M. Tobisa, Y. Nakano, N. Ohba, Y. Yano and Y. Masuda 2003a A symbolic representation of field-forage-ruminant relationships using polar form on the complex plane. J. Fac. Agr., Kyushu Univ., 47: 359-366

Shimojo, M., Y. Asano, K. Ikeda, R. Ishiwaka, T. Shao, H. Sato, M. Tobisa, Y. Nakano, N. Ohba, Y. Yano and Y. Masuda 2003b Complex representation of field-forage-ruminant relationships using symmetric properties of Euler's formula. J. Fac. Agr., Kyushu Univ., 47: 367-372

Shimojo, M., K. Ikeda, Y. Asano, R. Ishiwaka, T. Shao, H. Sato, M. Tobisa, Y. Nakano, N. Ohba, Y. Yano and Y. Masuda 2003c Exponential functions with base $e$ in growth analysis and deriving them from rotations of axes of time described using Euler's formula. J. Fac. Agr., Kyushu Univ., 48: 65-69

Shimojo, M., Y. Asano, K. Ikeda, R. Ishiwaka, T. Shao, H. Sato, M. Tobisa, Y. Nakano, N. Ohba, Y. Yano and Y. Masuda 2003d A Stereographic representation of Euler's formula to show spirals and topological similarities to micro-structures in ruminants and forages. J. Fac. Agr., Kyushu Univ., 48: 71-75

Yoshida, T. 2000 Emotion for imaginary number. Tokai University Press, Tokyo. (Written in Japanese) 